\title{
Magnetic order and spin dynamics in the proximity of a ferromagnetic quantum critical point: A $\mu$ SR study of $\mathrm{YbNi}_{4} \mathrm{P}_{2}$
}

\author{
J. Spehling, ${ }^{1}$ M. Günther, ${ }^{1}$ C. Krellner,,${ }^{2,3}$ N. Yèche, ${ }^{1}$ H. Luetkens,${ }^{4}$ C. Baines, ${ }^{4}$ C. Geibel,${ }^{2}$ and H.-H. Klauss ${ }^{1, *}$ \\ ${ }^{1}$ Institute for Solid State Physics, TU Dresden, D-01069 Dresden, Germany \\ ${ }^{2}$ Max Planck Institute for Chemical Physics of Solids, D-01187 Dresden, Germany \\ ${ }^{3}$ Cavendish Laboratory, University of Cambridge, J. J. Thomson Avenue, Cambridge CB3 OHE, United Kingdom \\ ${ }^{4}$ Laboratory for Muon-Spin Spectroscopy, Paul-Scherrer-Institute, CH-5232 Villigen, Switzerland \\ (Received 9 December 2011; revised manuscript received 29 February 2012; published 12 April 2012)
}

\begin{abstract}
The local $4 f$-electronic spin dynamics and magnetic order in $\mathrm{YbNi}_{4} \mathrm{P}_{2}$ were studied by means of muon-spin relaxation measurements. Zero-field muon-spin relaxation proves static magnetic order with a strongly reduced ordered $\mathrm{Yb}^{3+}$ moment of $(2.5-4.6) \times 10^{-2} \mu_{B}$, below $T_{C}=140 \mathrm{mK}$. Above $T_{C}$, the muon-spin polarization $P(t, B)$ is dominated by quasihomogeneous spin fluctuations and exhibits a time-field scaling relation $P(t, B)=$ $P\left(t / B^{\gamma}\right)$, indicating cooperative critical spin dynamics in the system. At $T=190 \mathrm{mK}$, slightly above $T_{C}, \gamma=$ 0.81(5), suggesting time-scale invariant power-law behavior for the dynamic electronic spin-spin autocorrelation function.
\end{abstract}

DOI: 10.1103/PhysRevB.85.140406

PACS number(s): 71.27.+a, 75.30.-m, 76.75.+i, 75.50.Cc

Lanthanide-based heavy-fermion (HF) systems are suitable model systems to study emergent phenomena at a quantum critical point (QCP), where collective quantum fluctuations trigger the system continuously from a magnetically ordered to a nonmagnetic ground state. ${ }^{1-6}$ However, despite intense research, to the best of our knowledge, no $4 f$-based material is known with a continuous ferromagnetic (FM) to paramagnetic (PM) quantum phase transition (QPT). The existence of such a QPT is also controversially discussed from a theoretical point of view. ${ }^{7-11}$

Recently, Krellner et al. suggested that the HF metal $\mathrm{YbNi}_{4} \mathrm{P}_{2}$ with a quasi-one-dimensional (1D) electronic structure exhibits FM quantum criticality above a low FM transition temperature $T_{C}=170 \mathrm{mK} .{ }^{12} \mathrm{YbNi}_{4} \mathrm{P}_{2}$ crystallizes in the tetragonal $\mathrm{ZrFe}_{4} \mathrm{Si}_{2}$ structure containing isolated chains of edge-connected $\mathrm{Ni}$ tetrahedra along the $c$ axis. The $\mathrm{Yb}$ atoms are located in the channels between these $\mathrm{Ni}$ tetrahedral chains. The reduced dimensionality in the $\mathrm{Yb}$ and Ni network and the geometrical frustration between neighboring $\mathrm{Yb}$ chains give rise to enhanced quantum spin fluctuations of the magnetic $\mathrm{Yb}^{3+}$ ions. In the PM state above $50 \mathrm{~K}$, the magnetic susceptibility shows Curie-Weiss behavior with an effective moment $\mu_{\mathrm{eff}}=4.52 \mu_{B}$ that is characteristic for magnetic $\mathrm{Yb}^{3+}$ ions. Analysis of the magnetic entropy reveals a Kondo energy scale of $T_{K} \approx 8 \mathrm{~K}$ for the crystal electric field ground-state doublet. The FM transition is evidenced by distinct anomalies in magnetic susceptibility, specific heat, and resistivity measurements. Low- $T$ magnetization measurements suggest an ordered FM moment of $m_{\text {ord }} \approx 0.05(4) \mu_{B}$. Pronounced non-Fermi-liquid (NFL) behavior is reflected by a stronger-than-logarithmic diverging Sommerfeld coefficient and a linear-in- $T$ resistivity state apparent in a $T$ range larger than a decade above $T_{C}$. In external magnetic fields, the NFL behavior is suppressed and FL behavior gradually recovers. Therefore, $\mathrm{YbNi}_{4} \mathrm{P}_{2}$ is considered as a clean system situated in the very close vicinity of a FM QCP, with FM quantum fluctuations dominating thermodynamic and transport quantities at $T>T_{C}$.
The present knowledge on $\mathrm{YbNi}_{4} \mathrm{P}_{2}$ is based on measurements of macroscopic magnetic, thermodynamic, and transport properties. The next step in a deeper investigation of this prospective FM quantum critical system is to get insight on a microscopic level. Besides the nature of the magnetic order, a central issue in the present context of critical behavior is the spin dynamics. Since in systems close to a QCP, the ordered moment is usually strongly reduced, muon-spin relaxation ( $\mu \mathrm{SR}$ ) has proven to be an extremely valuable technique to collect appropriate information. ${ }^{13-15}$

Here, we present $\mu \mathrm{SR}$ experiments on polycrystalline $\mathrm{YbNi}_{4} \mathrm{P}_{2}$, providing microscopic evidence for static magnetism at $T \leqslant T_{C} \approx 140 \mathrm{mK}$ with an ordered moment of $m_{\text {ord }}=(2.5-4.6) \times 10^{-2} \mu_{B} / \mathrm{Yb}$, depending on the assumed muon site. Above $T_{C}$, the muon-spin polarization $P(t)$ obeys the time-field scaling relation $P(t)=P\left(t / B^{0.81(5)}\right)$, indicating cooperative and critical spin dynamics.

In a $\mu$ SR experiment positive spin-polarized muons are implanted into the sample, and the subsequent time evolution of the muon-spin polarization is monitored by detecting the asymmetric spatial distribution of positrons emitted from the muon decay. ${ }^{16} \mu \mathrm{SR}$ in longitudinal applied magnetic fields is dominated by $\mathrm{Yb}-4 f$ electronic spin fluctuations that couple to the implanted muons. The $\mu \mathrm{SR}$ experiments on $\mathrm{YbNi}_{4} \mathrm{P}_{2}$ in zero field (ZF) and longitudinal (LF) applied field-with respect to the initial muon spin polarization-were performed on the $\pi \mathrm{M} 3$ beam line at the Swiss Muon Source at the Paul-ScherrerInstitut, Switzerland. The sample was prepared by crushing $\sim 270 \mathrm{mg}$ of single-crystalline material, grown in a self-flux at $1400^{\circ} \mathrm{C}$ in a closed Tantal crucible, and characterized by powder $\mathrm{x}$-ray diffraction experiments, proving the absence of any foreign phases. Detailed low- $T$ measurements on polycrystalline $\mathrm{YbNi}_{4} \mathrm{P}_{2}$ were reported elsewhere. ${ }^{12}$

Figure 1(a) displays typical time dependencies of the ZF muon-spin polarization $P(t)$ in $\mathrm{YbNi}_{4} \mathrm{P}_{2}$ at representative temperatures above and below $T_{C}$. A finite $T$-independent background signal due to muons that stopped in a $\mathrm{Ag}$ sample holder (signal fraction $\approx 50 \%$ ) was taken into account. At $T \geqslant 160 \mathrm{mK}$, an exponential muon-spin relaxation is 

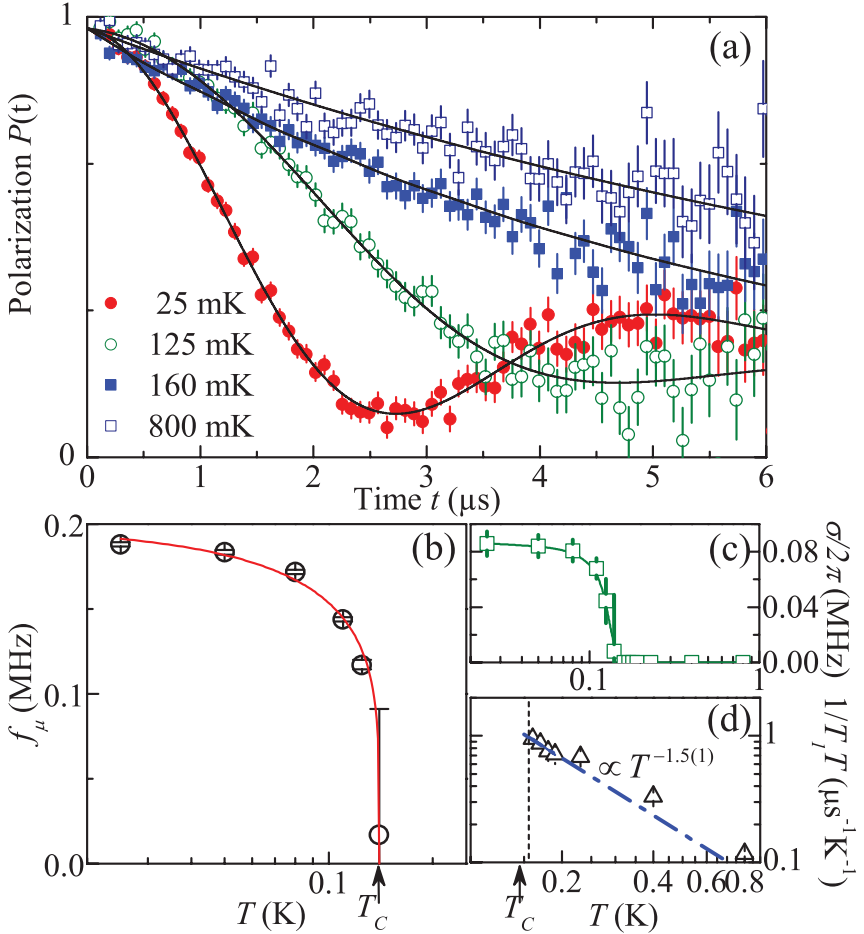

FIG. 1. (Color online) (a) Corrected muon-spin polarization $P(t)$ at $\mathrm{ZF}$ for representative $T$ above and below $T_{C} \approx 140 \mathrm{mK}$. At $T \leqslant T_{C}$, solid lines are fitting curves according to Eq. (1). (b) $T$ dependence of the ZF $\mu$ SR frequency $f_{\mu}(T)$. The solid line is a fit to the phenomenological function $f_{\mu}=f_{\mu}(0)\left(1-\frac{T}{T_{C}}\right)^{n}$. (c) $T$ dependence of the ZF static internal field distribution $\sigma$ in Eq. (1). The solid line is a guide to the eye. (d) $T$ dependence of $1 / T_{1} T$ in the $\mathrm{PM}$ regime. The line describes power-law behavior as $\frac{1}{T_{1} T} \propto T^{-1.5}$.

associated with fast fluctuating paramagnetic electron spins with a relaxation rate $\lambda(160 \mathrm{mK}) \approx 0.152(2) \mu \mathrm{s}^{-1}$. Note that dense static nuclear dipole moments would give rise to a weak Gaussian relaxation in the PM regime. While cooling through $T_{C}$, an additional magnetic relaxation mechanism is apparent, strongly increasing with lowering $T$. Below $T_{C}$, a low-frequency oscillation with a Gaussian relaxation of the muon-spin polarization is observed, indicating magnetic ordering of weak electronic $\mathrm{Yb}^{3+}$ moments. The muon-spin asymmetry data in the FM regime can be described best using the functional form ${ }^{17,18}$

$$
P(t)=\frac{1}{3}+\frac{2}{3}\left[\cos \left(2 \pi f_{\mu} t\right)-\frac{\sigma^{2} t}{2 \pi f_{\mu}} \sin \left(2 \pi f_{\mu} t\right)\right] e^{-\frac{1}{2} \sigma^{2} t^{2}},
$$

where $f_{\mu}$ and $\sigma$ are the muon-spin precession frequency and the Gaussian field width, respectively. The $2 / 3$ oscillating and the $1 / 3$ nonoscillating terms originate from the spatial averaging in polycrystalline samples, where $2 / 3(1 / 3)$ of the internal magnetic field components are directed perpendicular (parallel) to the initial muon spin, causing a precession (no precession) of the muon spin. The observation of a $2 / 3$ and $1 / 3$ signal fraction below $T_{C}$ implies dense magnetic moments and proof that $100 \%$ of the sample volume shows static magnetic order. The latter is supported by LF- $\mu \mathrm{SR}$ measurements, as discussed in detail below. In the limit $2 \pi f_{\mu} \gg \sigma$, Eq. (1) becomes a Gaussian damped cosine function. For $2 \pi f_{\mu} \rightarrow 0$, close to the magnetic transition, Eq. (1) is equivalent to the Gaussian Kubo-Toyabe (GKT) function, ${ }^{19}$ which describes a muon-spin relaxation due to a static Gaussian field distribution centered around $B_{\text {local }}=0 . \mathrm{ZF}-\mu \mathrm{SR}$ on the antiferromagnetically ordered system $\mathrm{YbRh}_{2} \mathrm{Si}_{2}$ (Ref. 14) reveals a similar crossover from a Lorentzian to a Gaussian damped $\mu \mathrm{SR}$ signal in the vicinity of the PM to magnetic phase transition, attributed to a transition from dynamic to static magnetism of magnetic $\mathrm{Yb}^{3+}$ moments.

For $\mathrm{YbNi}_{4} \mathrm{P}_{2}$ a finite $\mu \mathrm{SR}$ frequency is clearly observed below $150 \mathrm{mK}$. From the measured frequency value $f_{\mu}=$ $0.188(1) \mathrm{MHz}$ at $20 \mathrm{mK}$ one can determine the internal local field at the muon site to $B_{\text {local }}=13.87 \mathrm{G}$ using $B_{\text {local }}=2 \pi f_{\mu} / \gamma_{\mu}$, with $\gamma_{\mu}=2 \pi \times 13.55 \mathrm{kHz} / \mathrm{G}$ as the muon gyromagnetic ratio. The local field $B_{\text {local }}$ as well as the local static field width $\Delta B_{\text {local }}=\sigma / \gamma_{\mu} \approx 6 \mathrm{G}$ are very small for conventional rare-earth magnets with large ordered moments. The fractional width $\Delta B_{\text {local }} / B_{\text {local }}$ of the spontaneous field distribution is $\sim 0.4$ at low $T$ and remains constant as $T \rightarrow T_{C}$, which is a reasonable value for a magnetically ordered HF system, as, e.g., in $\mathrm{CeRhIn}_{5}, \Delta B_{\text {local }} / B_{\text {local }}=0.5$ is observed. ${ }^{20}$ Thus, the local field distribution is nearly uniform and homogeneous in the FM regime. The spontaneous muon-spin precession and the Gaussian shape of the internal field distribution below $T_{C}$ arise from a dense system of weak magnetic moments with small, static magnetic inhomogeneities. The presence of a finite $B_{\text {local }} \neq 0$ proves coherent magnetic order.

$\mathrm{ZF}-\mu \mathrm{SR}$ allows a precise determination of the $T$ dependence of the magnetic order parameter, which is proportional to the measured $\mu \mathrm{SR}$ frequency $f_{\mu}$. The $T$ dependence of $f_{\mu}$ and $\sigma$ is shown in Figs. 1(b) and 1(c). For $T \leqslant$ $140 \mathrm{mK}$, both observables exhibit a continuous increase. The $T$ dependence of $f_{\mu}$ can be fit to the phenomenological function $f_{\mu}=f_{\mu}(0)\left(1-\frac{T}{T_{C}}\right)^{n}$ for $T<T_{C}$ with $n=0.208 \pm$ $0.02, f_{\mu}(T)=0.199(3) \quad{ }^{T_{C}} \mathrm{MHz}$, and $T_{C}=140(2) \mathrm{mK}$. The value of the effective critical exponent $n$, describing the critical behavior close to $T_{C}$, is between $n=0.125$ and 0.325 , which are theoretically expected for two-dimensional (2D) and isotropic three-dimensional (3D) Ising magnets, respectively. This is not in contradiction with the claim of a quasi-1D system. In such a system, the weak interchain coupling results in an evolution from a 1D behavior at high $T$ to a $2 \mathrm{D}$ Ising or 3D behavior at low $T$, which is intimately linked with (and is a prerequisite for) the long-range ordering at finite $T$. The low data point density between $0.6 \leqslant \frac{T}{T_{C}} \leqslant 1$, however, precludes the determination of the precise critical exponent. The obtained value for $T_{C}$ agrees well with the value found in specific-heat measurements on these single crystals. ${ }^{21}$

For all examined $T \leqslant T_{C}$, the sample signal is analyzed with a well-defined single $f_{\mu}$ and $\sigma$, signaling that the magnetic order is a bulk effect and that only one dominant muon stopping site is present. In general, for the determination of the muon stopping site(s) it is important to deduce the hyperfine coupling constant. One way to find potential muon sites is to compare calculated and measured quantities for the local field $B_{\text {local }}$ at the muon site. The muon preferentially settles at tetrahedra or octahedra interstitial crystallographic sites. From simple symmetry arguments the 
most probable muon stopping sites, using Wyckoff's notation, are $4 f(1 / 4,1 / 4,0), 8 j(1 / 4,1 / 4,1 / 4), 4 f(1 / 4,1 / 4,1 / 2)$, $8 i(1 / 4,1 / 2,1 / 2), 4 c(1 / 2,0,0), 4 c(1 / 2,0,1 / 2), 2 b(0,0,1 / 2)$, and $2 a(1 / 2,1 / 2,1 / 2)$. For a particular FM structure with the magnetic $\mathrm{Yb}^{3+}$ moments aligned within the $a-b$ plane and a dominant $4 f-\mu$ dipolar interaction, one can determine the expected internal field values for the proposed sites. Our lattice sum calculations reveal that only at the $4 c(1 / 2,0,1 / 2)$ site and $8 i(1 / 4,1 / 2,1 / 2)$ site a local field $B_{\text {local }}$ of the measured absolute magnitude is found. For the $4 c$ and $8 i$ sites the measured local field of $B_{\text {local }}=13.87 \mathrm{G}$ corresponds to a static ordered moment of the Yb ions of $m_{\text {ord }}=0.046 \mu_{B}$ and $0.025 \mu_{B}$, respectively. Both values are in good agreement with the value deduced from recent magnetization measurements. ${ }^{12}$

The temperature dependence of the exponential relaxation rate $\lambda_{L}=\frac{1}{T_{1}}$, observed above $T_{C}$, is plotted in Fig. 1(d) on a $\log$ - $\log$ scale as $\frac{1}{T_{1} T}$. Cooling down from $800 \mathrm{mK}, \frac{1}{T_{1} T}$ exhibits power-law behavior according to $\frac{1}{T_{1} T} \propto T^{-1.40(6)}$. At $T \leqslant 190 \mathrm{mK}$, the power-law behavior in $\frac{1}{T_{1} T}$ persists in the PM regime down to $T_{C}$, however, with a slight change of the critical exponent, i.e., $\frac{1}{T_{1} T} \propto T^{-1.5(1)}$ (dashed-dotted line). The observed $\frac{1}{T_{1} T}$ behavior is close to the $T^{-4 / 3}$ temperature dependence predicted by the self-consistent renormalization (SCR) theory for a system close to a 3D ferromagnetic QCP. ${ }^{22}$ There is no prediction for an itinerant quasi-1D system in the $T$ range between the exchange energy scale and ordering temperature. For an isolating ferromagnetic quasi-1D spin chain the $T$ dependence of the relaxation rate above $T_{C}$ depends strongly on the details of the interactions-see, e.g., Ref. 23.

LF- $\mu$ SR experiments allow to separate the dynamic contribution to the relaxation of the muon-spin polarization. Investigations of the low- $T$ muon-spin dynamics yield additional information about the origin of the NFL behavior in $\mathrm{YbNi}_{4} \mathrm{P}_{2}$. Figure 2 displays the muon-spin asymmetry function $P(t)$ at $T=20 \mathrm{mK}$ for different applied LFs. The muon-spin relaxation is completely suppressed in an applied field $B_{\mathrm{LF}} \approx 300 \mathrm{G}$, demonstrating that the internal field distribution is static in nature. However, the observed decoupling cannot be described accurately by a standard muon asymmetry function that considers an internal field distribution

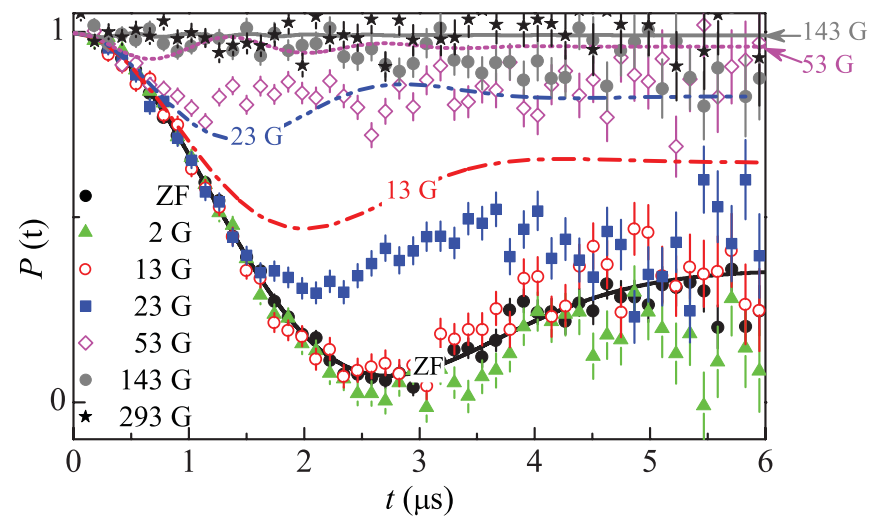

FIG. 2. (Color online) Corrected muon-spin polarization at $T=$ $20 \mathrm{mK}$ and various longitudinal magnetic fields $B_{\mathrm{LF}}$. The lines represent theoretical depolarization curves for the static GKT function in corresponding longitudinal fields.

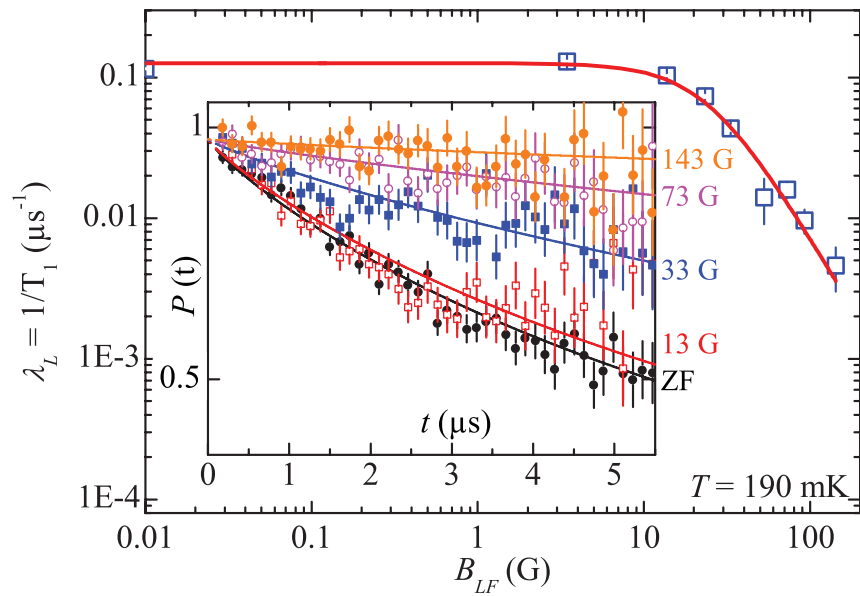

FIG. 3. (Color online) Main panel: Field dependence of the dynamic muon-spin relaxation rate $\lambda_{L}$. The solid curve represents a Redfield fit. For display reasons, the $\mathrm{ZF}$ value is set at $B_{\mathrm{LF}}=$ $0.01 \mathrm{G}$. Inset: Field dependence of the corrected muon-spin polarization $P(t)$ at $T=190 \mathrm{mK}$.

which is symmetric around $B_{\text {local }}=0$. For comparison, Fig. 2 shows theoretical depolarization curves for the static GKT function in the corresponding longitudinal magnetic fields. This supports the ZF data, i.e., the observation of a broad field distribution centered around a finite but small internal field $B_{\text {local }}(20 \mathrm{mK}) \approx 13.87 \mathrm{G}$ in the FM phase. Finally, when $B_{\mathrm{LF}} \gg B_{\text {local }}$, the muon-spin relaxation is decoupled from the static $B_{\text {local }}$ as observed for $B_{\mathrm{LF}} \geqslant 23 \mathrm{G}$.

At $T>T_{C}$, the field dependence of the muon-spin relaxation probes the Fourier transform of the dynamic spin-spin autocorrelation function $q(t)=\left\langle\mathbf{S}_{i}(t) \cdot \mathbf{S}_{i}(0)\right\rangle$, which exhibits exponential behavior for homogeneous systems and power-law (or cutoff power-law) or stretched exponential behavior for inhomogeneous systems. The inset of Fig. 3 displays the muon-spin polarization $P(t)$ at $T=190 \mathrm{mK}$, both in magnetic LF between 13 and $143 \mathrm{G}$ and $\mathrm{ZF}$. The relaxation rate $\lambda_{L}$ is reduced with increasing field. The field dependence of $\lambda_{L}$ is given in the main panel of Fig. 3. It shows nearly no field dependence for magnetic fields of less than $\sim 13 \mathrm{G}$, but varies more strongly, as $H^{-\kappa}$ with $\kappa \approx 0.79(7)$, for higher fields. From the field dependence of $\lambda_{L}$, the spin autocorrelation time $\tau_{c}$ can be estimated using the Redfield formalism for $\lambda_{L}\left(B_{\mathrm{LF}}\right)=\left(2 \gamma_{\mu}^{2}\left\langle B_{\mathrm{fluc}}^{2}\right\rangle \tau_{c}\right) /\left[1+\left(\gamma_{\mu}^{2} B_{\mathrm{LF}}^{2} \tau_{c}^{2}\right)\right]$ considering $\tau_{c}$ as independent of the applied field $B_{\mathrm{LF}}$. Here, $B_{\text {fluc }}(t)$ describes the time-varying local magnetic field at the muon site due to fluctuations of neighboring $\mathrm{Yb}^{3+}$ moments, with a local time-averaged second moment $\Delta^{2}=\gamma_{\mu}^{2}\left\langle B_{\text {fluc }}^{2}\right\rangle$ and a single fluctuation time $\tau_{c}$. For $\hbar \omega \ll k_{B} T$ ( $\omega$ giving the spin fluctuation rate), the fluctuation-dissipation theorem ${ }^{24}$ relates $\tau_{c}$ to the imaginary component of the local $q$-independent $f$-electron dynamic susceptibility, i.e., $\tau_{c}(B)=\left(k_{B} T\right)\left[\chi^{\prime \prime}(\omega) / \omega\right]$. The fit to the data (solid curve in the main panel of Fig. 3) yields $\Delta^{2} \approx 0.1(\mathrm{MHz})$ and $\tau_{c} \approx 6 \times 10^{-7} \mathrm{~s}$, the latter value nearly three orders of magnitude larger than the one obtained for $\mathrm{YbRh}_{2} \mathrm{Si}_{2}$ at $T=20 \mathrm{mK},{ }^{14}$ suggesting very slow critical fluctuations.

The $\mu$ SR time spectra in Fig. 3 are well described with a stretched exponential relaxation function of the form 


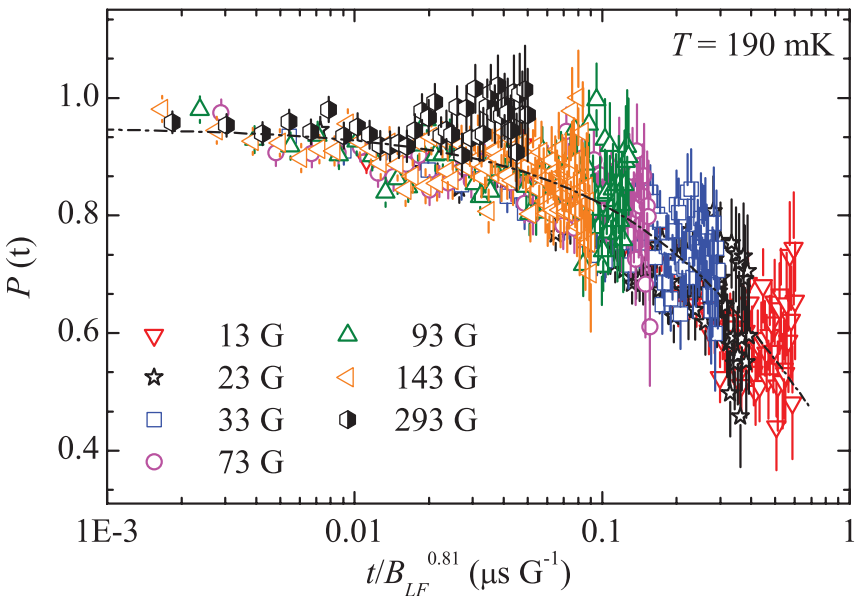

FIG. 4. (Color online) Corrected muon decay asymmetry at $T=190 \mathrm{mK}$ for various magnetic fields as function of the scaling variable $t / B_{\mathrm{LF}}^{0.81}$. The dashed-dotted line is a fit of the $13-\mathrm{G}$ data with $P(t) / P(0)=\exp \left[-\lambda_{L} t\right]^{-0.9}$

$P(t)=P(0) \exp \left[-(\lambda t)^{\beta}\right]$. An exponent of $\beta \approx 0.9$ shows that the relaxation rate is nearly uniform throughout the sample, indicating that $\mathrm{YbNi}_{4} \mathrm{P}_{2}$ exhibits quasihomogeneous spin fluctuations for $T \ll T_{K}$. The spin dynamics is characterized by a narrow distribution of correlation times $(\beta=1$ corresponds to one single correlation time). Thus, disorder-driven theories, including Kondo disorder ${ }^{25,26}$ and the Griffith phase scenario ${ }^{27}$ as primary mechanisms for the observed NFL behavior, can be ruled out. It further implies that the crystalline disorder in $\mathrm{YbNi}_{4} \mathrm{P}_{2}$ is quite small, which is consistent with a small residual resistivity $\left(\rho_{0} \sim 2.4 \mu \Omega \mathrm{cm}\right)$ and the stoichiometric occupation of the crystallographic lattice sites revealed by the $\mathrm{X}$-ray structure refinement. ${ }^{12}$

A sensitive test to identify power-law or stretched exponential behavior of $q(t)$ is a time-field scaling analysis of the muonspin relaxation function. In both cases a specific time-field scaling can be found, i.e., the muon-spin relaxation function $P\left(t, B_{\mathrm{LF}}\right)$ obeys the scaling relation $P\left(t, B_{\mathrm{LF}}\right)=P\left(t / B_{\mathrm{LF}}^{\gamma}\right)$. This relation applies only in the asymptotic strong field limit, i.e., as long as $2 \pi f_{\mu}=\gamma_{\mu} B_{\mathrm{LF}} \gg \lambda_{L}{ }^{28}$ If time-field scaling is obeyed, a plot of $P\left(t, B_{\mathrm{LF}}\right)$ versus $t / B_{\mathrm{LF}}^{\gamma}$ at $T>T_{C}$ will be universal for the correct choice of $\gamma$, and distinguishes between power-law $(\gamma<1)$ and stretched exponential $(\gamma \geqslant 1)$ correlations. For small $B_{\mathrm{LF}}$, the field dependence is expected to be due to the change of $f_{\mu}$ rather than an effect of field on $q(t)$. A breakdown of time-field scaling would occur for high fields where $q(t)$ is directly effected by the applied fields. Figure 4 shows the same asymmetry data, as displayed in Fig. 3, as functions of the scaling variable $t / B_{\mathrm{LF}}^{\gamma}$. For $\gamma=0.81(5)$ the data scale well over $\sim 2.5$ orders of magnitude in $t / B_{\mathrm{LF}}^{\gamma}$ and for all fields between 13 and $143 \mathrm{G}$, except for $293 \mathrm{G}$. Here, at large $t$, the data fall above the low-field scaling curve. Fields $\mu_{B} B_{\mathrm{LF}} \geqslant k_{B} T$ (with $k_{B}=$ Boltzmanns constant) would be expected to affect the spin dynamics. The scaling exponent $\gamma=0.81(5)<1$ implies that within the $\mu$ SR frequency range, the spin-spin correlation function $q(t)$ is approximated by a power law (or a cutoff power law) rather than a stretched exponential or exponential, ${ }^{28}$ consistent with the Redfield analysis. The power law is time-scale invariant and dynamical modulations should therefore be observable in any time window. The obtained time-field scaling of the relaxation data is a signature of slow homogeneous spin dynamics. It strongly indicates that the critical slowing down of spin fluctuations at the magnetic phase transition occurs cooperatively throughout the sample. In stoichiometric, homogeneous NFL systems such behavior may arise from the effect of disorder on quantum critical fluctuations inherent to a QCP. This is suggested for the NFL compound $\mathrm{YbRh}_{2} \mathrm{Si}_{2} .{ }^{14,15}$

In conclusion, $\mathrm{ZF}-\mu \mathrm{SR}$ in the stoichiometric NFL compound $\mathrm{YbNi}_{4} \mathrm{P}_{2}$ clearly proves static magnetic ordering of strongly reduced $\mathrm{Yb}^{3+}$ moments below $T_{C}=140 \mathrm{mK}$. Above $T_{C}$, the muon-spin polarization $P(t)$ obeys the time-field scaling relation $P(t)=P\left(t / B^{0.81(5)}\right)$ for applied magnetic fields $B$ between 13 and $143 \mathrm{G}$, indicating cooperative and critical spin dynamics. Power-law behavior of the dynamic spin-spin autocorrelation function is implied by the observation of $\gamma<1 .^{28}$ The LF- $\mu$ SR results suggest that the NFL behavior observed at $T>T_{C}$ is induced by quasihomogeneous critical spin fluctuations.

We acknowledge with thanks the help of A. Amato and the PSI accelerator crew as well as financial support by the German Science Foundation (DFG) in the framework of the priority program 1458, Grant No. KL1086/10-1. *h.klauss@physik.tu-dresden.de

${ }^{1}$ Focus issue on quantum phase transitions, Nat. Phys. 4, 167 (2008).

${ }^{2}$ N. D. Mathur, F. M. Grosche, S. R. Julian, I. R. Walker, D. M. Freye, R. K. W. Haselwimmer, and G. G. Lonzarich, Nature (London) 394, 39 (1998).

${ }^{3}$ G. R. Stewart, Rev. Mod. Phys. 73, 797 (2001); 78, 743 (2006).

${ }^{4}$ T. Park, F. Ronning, H. Q. Yuan, M. B. Salamon, R. Movshovich, J. L. Sarrao, and J. D. Thompson, Nature (London) 440, 65 (2006).

${ }^{5}$ S. Friedemann, T. Westerkamp, M. Brando, N. Oeschler, S. Wirth, P. Gegenwart, C. Krellner, C. Geibel, and F. Steglich, Nat. Phys. 5, 465 (2009).
${ }^{6}$ O. Stockert, J. Arndt, E. Faulhaber, C. Geibel, H. S. Jeevan, S. Kirchner, M. Loewenhaupt, K. Schmalzl, W. Schmidt, Q. Si, and F. Steglich, Nat. Phys. 7, 119 (2011).

${ }^{7}$ T. R. Kirkpatrick and D. Belitz, Phys. Rev. B 67, 024419 (2003).

${ }^{8}$ G. J. Conduit, A. G. Green, and B. D. Simons, Phys. Rev. Lett. 103, 207201 (2009).

${ }^{9}$ S. J. Yamamoto and Q. Si, Proc. Natl. Acad. Sci. USA 107, 15704 (2010).

${ }^{10}$ Una Karahasanovic, Frank Krüger, and Andrew G. Green, Phys. Rev. B 85, 165111 (2012).

${ }^{11}$ R. Peters, N. Kawakami, and T. Pruschke, Phys. Rev. Lett. 108, 086402 (2012). 
${ }^{12}$ C. Krellner, S. Lausberg, A. Steppke, M. Brando, L. Pedrero, H. Pfau, S. Tencè, H. Rosner, F. Steglich, and C. Geibel, New J. Phys. 13, 103014 (2011).

${ }^{13}$ D. E. MacLaughlin, O. O. Bernal, R. H. Heffner, G. J. Nieuwenhuys, M. S. Rose, J. E. Sonier, B. Andraka, R. Chau, and M. B. Maple, Phys. Rev. Lett. 87, 066402 (2001).

${ }^{14}$ K. Ishida, D. E. MacLaughlin, B.-L. Young, K. Okamoto, Y. Kawasaki, Y. Kitaoka, G. J. Nieuwenhuys, R. H. Heffner, O. O. Bernal, W. Higemoto, A. Koda, R. Kadono, O. Trovarelli, C. Geibel, and F. Steglich, Phys. Rev. B 68, 184401 (2003).

${ }^{15}$ D. E. MacLaughlin, R. H. Heffner, O. O. Bernal, K. Ishida, J. E. Sonier, G. J. Nieuwenhuys, M. B. Maple, and G. R. Stewart, J. Phys.: Condens. Matter 16, 4470 (2004), and references therein.

${ }^{16}$ A. Schenck, Muon Spin Rotation Spectroscopy: Principles and Applications in Solid State Physics (Hilger, Bristol, 1985).

${ }^{17}$ S. G. Barsov et al., Hyperfine Interact. 64, 415 (1990).

${ }^{18}$ E. I. Kornilov and V. Yu. Pomjakushin, Phys. Lett. A 153, 364 (1991).
${ }^{19}$ R. S. Hayano, Y. J. Uemura, J. Imazato, N. Nishida, T. Yamazaki, and R. Kubo, Phys. Rev. B 20, 850 (1979).

${ }^{20}$ A. Schenck, D. Andreica, F. N. Gygax, D. Aoki, and Y. Ōnuki, Phys. Rev. B 66, 144404 (2002).

${ }^{21}$ A. Steppke (private communication).

${ }^{22}$ T. Moriya and T. Takimoto, J. Phys. Soc. Jpn. 64, 960 (1995); A. Ishigaki and T. Moriya, ibid. 65, 3402 (1996); T. Misawa, Y. Yamaji, and M. Imada, ibid. 78, 084707 (2009).

${ }^{23}$ M. Sato, T. Hikihara, and T. Momoi, Phys. Rev. B 83, 064405 (2011).

${ }^{24}$ J. S. Toll, Phys. Rev. 104, 1760 (1956).

${ }^{25}$ E. Miranda, V. Dobrosavljevic, and G. Kotliar, J. Phys.: Condens. Matter 8, 9871 (1996).

${ }^{26}$ E. Miranda, V. Dobrosavljevic, and G. Kotliar, Phys. Rev. Lett. 78, 290 (1997).

${ }^{27}$ A. H. Castro Neto, G. Castilla, and B. A. Jones, Phys. Rev. Lett. 81, 3531 (1998).

${ }^{28}$ A. Keren, P. Mendels, I. A. Campbell, and J. Lord, Phys. Rev. Lett. 77, 1386 (1996). 